\title{
Stage IB Endometrioid Type Endometrial Cancer: The Role of Lymphadenectomy and Adjuvant Radiation Therapy
}

\author{
Osman TURKMEN', Tolga TASCI', Derman BASARAN', Gunsu COMERT KIMYON', \\ Alper KARALOK ${ }^{1}$, Isin UREYEN ${ }^{1}$, Taner TURAN ${ }^{1}$
}

Ankara, Turkey

\begin{abstract}
OBJECTIVE: Both performances of lymphadenectomy and benefit of adding adjuvant radiotherapy are controversial for patients with International Federation of Gynecology and Obstetrics stage IB endometrioid type endometrial cancer. We aimed to identify the role of lymphadenectomy and adjuvant radiation therapy as well as clinicopathological prognostic factors for this group of patients.
\end{abstract}

STUDY DESIGN: Records of all patients $(n=132)$ with stage IB endometrioid endometrial cancer who were referred to or treated in our institution between Jan 1992 and Dec 2013 were retrospectively reviewed. Cox Proportional Hazard Regression Analysis was used to determine the effects of lymphadenectomy and adjuvant radiation as well as other clinicopathological factors on disease free survival and overall survival.

RESULTS: Mean age was 59.9 years (range, 45-82). Lymphadenectomy didn't perform in 36 (27.3\%) patients and $23(17.4 \%)$ patients did not have any kind of adjuvant treatment. Mean lymph node count was 18.8 (range, 3-67). Federation of Gynecology and Obstetrics grade, lymphovascular space invasion, lymphadenectomy, receiving adjuvant treatment and type of received adjuvant therapy were not associated with disease free survival and overall survival for the entire cohort. In a subgroup of patients with grade1\&2 tumor, 5-year disease free survival rates were $80 \%$ and $50 \%(p=0.4)$, respectively and overall survival rates were $94.8 \%$ and $93.8 \%(p=0.2)$, respectively for patients who had or didn't have adjuvant radiotherapy. While performance of lymphadenectomy was not significantly associated with disease free survival in this subgroup $(p=0.56)$, this association was statistically significant for overall survival $(97.9 \%$ vs. $86.4 \%,(p=0.04)$.

CONCLUSION: Benefit of adjuvant radiotherapy in regard to prevention of recurrence needs to be confirmed by further studies. Lymphadenectomy had a survival benefit for patients with myometrial invasion greater than a half of myometrial thickness.

Keywords: Endometrial cancer, Early stage, Lymphadenectomy, Adjuvant therapy

Gynecol Obstet Reprod Med 2018;24(3):156-161

\author{
${ }^{1}$ University of Health Sciences Department of Gynecologic Oncology, \\ Etlik ZubeydeHanim Women's Health Teaching and Research Hospital, \\ Ankara \\ Address of Correspondence: Osman Turkmen \\ University of Health Sciences \\ Department of Gynecologic Oncology \\ Etlik Zubeyde Hanım Women's Health \\ Teaching and Research Hospital \\ 06010 Ankara, Turkey \\ osmanturkmen312@gmail.com \\ Submitted for Publication: 25.04.2017 \\ Accepted for Publication: $\quad 16.08 .2017$
}

\begin{tabular}{|c|c|}
\hline & Access this article online \\
\hline $\begin{array}{c}\text { Quick Response Code: } \\
\text { Website: www.gorm.com.tr } \\
\text { info@gorm.com.tr }\end{array}$ \\
\cline { 2 - 3 }
\end{tabular}

How to cite this article: Turkmen O. Tasci T. Basaran D. Comert Kimyon G. Karalok A. Ureyen I. Turan T. Stage IB Endometrioid Type Endometrial Cancer: The Role of Lymphadenectomy and Adjuvant Radiation Therapy. Gynecol Obstet Reprod Med 2018;24(3):156-161

\section{Introduction}

Although endometrial cancer (EC) is the most common gynecologic malignancy in the developed countries and the majority of cases are diagnosed at an early stage, both proper surgical approach and the need for adjuvant treatment are still being debated. Standard surgery consists of total hysterectomy and bilateral salpingo-oophorectomy. The performance of extensive staging surgery which includes pelvic and para-aortic lymphadenectomy and omentectomy shows wide variations between the centers worldwide (1-3). Tumor type, grade, tumor size, and depth of myometrial invasion (MI) are key prognostic factors for lymph node metastasis and recurrence and are used to assess the need for lymphadenectomy as well as adjuvant radiation treatment $(4,5)$. The recommendation of the International Federation of Gynecology and Obstetrics (FIGO) which was revised in 2009 has been adequate surgical staging in EC since 1988 (6). However, the survival benefit of systematic lymphadenectomy in early stage EC is not clear (5, 7-9) and lymphadenectomy procedures are associated with 
morbidity including; blood loss, vascular injury, longer operating times and prolonged postoperative hospital stays (1012). On the other hand, surgical staging may allow the identification of patients with documented lymphatic tumor status and potentially reduce the morbidity related to unnecessary radiation therapy by selecting the group of patients in whom adjuvant therapy can be omitted (11).

2009 FIGO classification system identifies 2 subgroups of stage I according to the depth of myometrial invasion (IA: MI $<50 \%$, IB: MI $\geq 50 \%$ ) (6). Specifically for endometrioid tumor type, the 5 -year survival rate is $90 \%$ in stage I patients (13) and no further treatment is recommended for patients with stage IA and grade $1 \& 2$ diseases (14). Both observations and adjuvant radiotherapy and/or chemotherapy can be chosen for the management of stage IB disease according to the presence or absence of poor prognostic factors (14-18).

In this paper, it's aimed to identify the role of lymphadenectomy and adjuvant radiation therapy as well as clinicopathological prognostic factors for stage IB endometrioid type endometrial cancer.

\section{Material and method}

All patients $(\mathrm{n}=132)$ with stage IB endometrioid endometrial cancer who were operated and followed at our institution or whose hysterectomy (not staging surgery) was made somewhere else and referred to us based on the diagnosis of EC, between Jan 1992 and Dec 2013, were identified. The data were abstracted from the electronic database of the institution and pathology reports. The staging was undertaken according to FIGO 2009 staging system. Lymphovascular space invasion (LVSI) was assessed on H\&E-stained slides, as carcinoma cells present within a definite endothelial-lined space, at a distance from the tumor. Pathologic examination of hysterectomy specimen was done on at least 4 sections. Pathology slides of those that were operated elsewhere were reviewed at the time of application to our institution.

In our institution lymphadenectomy decision for apparently uterine confined EC is made routinely based on the frozen/section results if pathologic examination of pre-operative probe curettage indicates grade 1 tumor. Total hysterectomy and bilateral salpingo-oophorectomy were performed and lymphadenectomy was omitted in those patients with all of the following: 1) MI less than $<50 \% 2$ ) tumor size smaller than $2 \mathrm{~cm}$ and 3) grade 1 tumor. Otherwise, patients undergo complete staging surgery that includes total hysterectomy and bilateral salpingo-oophorectomy as well as pelvic/para-aortic lymphadenectomy and omental biopsy. However, lymphadenectomy was omitted in some patients because; 1) operation was made somewhere else as incomplete staging surgery and the patient refused a repeat operation 2) frozen/section revealed a stage IA disease but final pathology indicated stage IB and the patient refused a repeat operation and 3) of discretion of the senior surgeon. Lymphadenectomy was performed in most of the patients by skeletonizing pelvic and para-aortic regions. Nevertheless, there were patients treated by the sampling of the suspicious lymph nodes at the discretion of the surgeon.

Adjuvant radiotherapy was given as vaginal brachytherapy or external beam radiotherapy or both. External beam pelvic radiotherapy was given to a total dose of 5000-5400 cGy in 200 cGy fractions and vaginal brachytherapy was given to a dose of 1500-2750 cGy in 550-750 cGy fractions.

The period from surgery to recurrence or last visit was defined as disease-free survival (DFS) and period from surgery to death or last visit was defined as overall survival (OS). Patients were followed-up every 3 months for 2 years after adjuvant therapy, every 6 months until the fifth year following treatment and yearly thereafter. In every visit, pelvic examination, abdominal ultrasonography, complete blood count and blood chemistry were performed. Chest X-ray was utilized yearly unless there is a clinical suspicion. Thoracic and/or abdominal computerized tomography was used when needed. Recurrences that occurred in true pelvis or vaginal cuff were defined as locoregional recurrence.

Data analyses were performed by using SPSS for Windows 11.5 package program. Categorical variables were analyzed with Kaplan-Meier Survival Analysis using LogRank Test to determine whether they had statistically significant effects on DFS and OS. Whether the continuous and discrete numeric variables had statistically significant effects on DFS and OS were calculated using univariate Cox Proportional Hazard Regression Analysis. $\mathrm{P}<0.05$ was considered statistically significant for the results. The institutional review board approved the research.

\section{Results}

The data of 132 patients with stage IB and endometrioid type histology were analyzed. The median age was 59.9 years (range, 45-82). The mean tumor size was $3.4 \mathrm{~cm}$ (range, 1.6-6 $\mathrm{cm}$ ). The mean lymph node count was 18.8 (range, 3-67). Out of 132 patients, $36(27.3 \%)$ had the only hysterectomy with bilateral salpingo-oophorectomy without lymphadenectomy and $23(17.4 \%)$ did not have any kind of adjuvant treatment. Distribution of patients by surgicopathological characteristics and treatment modalities are summarized in table 1 . The median follow-up period was 59.5 months (range, 3-178). That patient with a follow-up period shorter than 3 months $(\mathrm{n}=11)$ was excluded from the survival analyses. Overall, 22 (18.2\%) patients had a recurrence and 7 patients died. Two of these 7 patients died in 2 months postoperatively so they were excluded from the survival analysis. Recurrences were locoregional in 9 patients whereas recurrence pattern was distant metastasis in 13 patients. The median recurrence time was 22.5 months. Five-year DFS and OS rates for the entire group were $76.6 \%$ and $94 \%$, respectively. 
Table 1: Surgicopathological characteristics and treatment modalities, $n=132$

\begin{tabular}{lc}
\hline Parameters & $\mathrm{n}(\%)$ \\
\hline FIGO Grade & $38(28.8)$ \\
Grade 1 & $73(55.3)$ \\
Grade 2 & $21(15.9)$ \\
$\quad$ Grade 3 & $80(60.6)$ \\
Lymphovascular space invasion & $52(36.4)$ \\
$\quad$ Negative & $36(27.3)$ \\
Positive & $96(72.7)$ \\
Lymphadenectomy & \\
No & $23(17.4)$ \\
Yes & $1(0.8)$ \\
Adjuvant treatment & $30(22.7)$ \\
No adjuvant treatment & $61(46.2)$ \\
Chemotherapy & $6(4.5)$ \\
Brachytherapy alone & $11(8.3)$ \\
External beam pelvic radiation alone & \\
Brachytherapy + External beam pelvic radiation & \\
Radiotherapy field N/A & \\
\hline
\end{tabular}

There was no clinicopathological factor or treatment modality associated with DFS and OS (Table 2). However, we observed that 5-year DFS rate was lower for patients who did not receive any kind of adjuvant treatment compared to those who had, although this difference was not statistically significant $(50 \%$ vs. $78.1 \%, \mathrm{p}=0.51)$.
Analysis of patients with grade 1, 2, 3 was made in respect to the association between the treatment modalities and survival. In all patients, adjuvant radiotherapy and type of adjuvant radiation were not associated with DFS and OS $(p=0.283$ and $\mathrm{p}=0.315$ respectively, for DFS and $\mathrm{p}=0.423$ and $\mathrm{p}=0.398$, respectively, for OS) (Table 3).

Table 2: Survival rate by clinicopathological factors and treatment modalities

\begin{tabular}{lcccc}
\hline Parameters & 5-year DFS (\%) & $p$ value & 5-year OS (\%) & $p$ value \\
\hline Age & 80.1 & 0.68 & 95.1 & 92.4 \\
$\quad \leq 60$ & 72.3 & & & 0.49 \\
$>60$ & & &
\end{tabular}

Tumor grade

Grade 1

84.1

94.7

Grade 2

75.1

0.4

93.9

0.82

Grade 3

66.2

92.9

Lymphovascular space invasion

$\begin{array}{llll}\text { Negative } & 74.8 & 9.1 \\ \text { Positive } & 79.2 & 0.95 & 94\end{array}$

Lymphadenectomy

No $\quad 74.5$

Yes $\quad 77.1$

0.95

87.7

96.8

Adjuvant treatment

No $\quad 50$

$\begin{array}{ll}\text { Yes } & 78.1\end{array}$

0.51

94.1

86

94.5

Radiotherapy received

BRT alone

100

EBRT alone

77.6

0.16

92.9

EBRT+BRT

50 
Table 3: The survival effects of lymphadenectomy and adjuvant treatment on patients with grade 1, 2,3 tumor

\begin{tabular}{|c|c|c|c|c|}
\hline Parameters & 5-year DFS (\%) & $p$ value & 5-year OS (\%) & $p$ value \\
\hline \multicolumn{5}{|c|}{ Lymphadenectomy } \\
\hline No & 74.8 & \multirow{2}{*}{0.95} & 85.3 & \multirow{2}{*}{0.08} \\
\hline Yes & 77.1 & & 95.8 & \\
\hline \multicolumn{5}{|c|}{ Adjuvant Radiotherapy } \\
\hline No & 47.7 & \multirow{2}{*}{0.283} & 97.8 & \multirow{2}{*}{0.423} \\
\hline Yes & 78.8 & & 89.7 & \\
\hline \multicolumn{5}{|c|}{ Field of adjuvant radiation } \\
\hline BRT alone & 75.4 & \multirow{3}{*}{0.315} & 100 & \multirow{3}{*}{0.398} \\
\hline EBRT alone & 75.8 & & 91.4 & \\
\hline $\mathrm{BRT}+\mathrm{EBRT}$ & 57.1 & & 83,3 & \\
\hline
\end{tabular}

DFS: Disease - free survival, OS: Overall survival, BRT: Brachytherapy, EBRT: External beam pelvic radiation

Similar to the entire group, patients who received adjuvant radiation had a lower recurrence compared to those who did not have adjuvant radiation $(47.7 \%$ vs. $78.8 \%)$, although this was not statistically significant. The performance of lymphadenectomy was not significantly associated with DFS and OS in all cohort ( $\mathrm{p}=0.95, \mathrm{p}=0.08$ ). However, in the subgroup analysis in patients with grade $1 \& 2$ tumor, lymphadenectomy was associated with better OS outcomes $(97.9 \%$ vs. $86.4 \%$, $\mathrm{p}=0.04$ ) (Figure 1). Furthermore, adjuvant treatment modalities were similar between the group of patients who had or did not have lymphadenectomy in this subgroup (Table 4).

There was no clinicopathological factor that had a $\mathrm{p}$ value of $<0.25$ except the presence or absence of lymphadenectomy, therefore multivariate analysis was not applied.

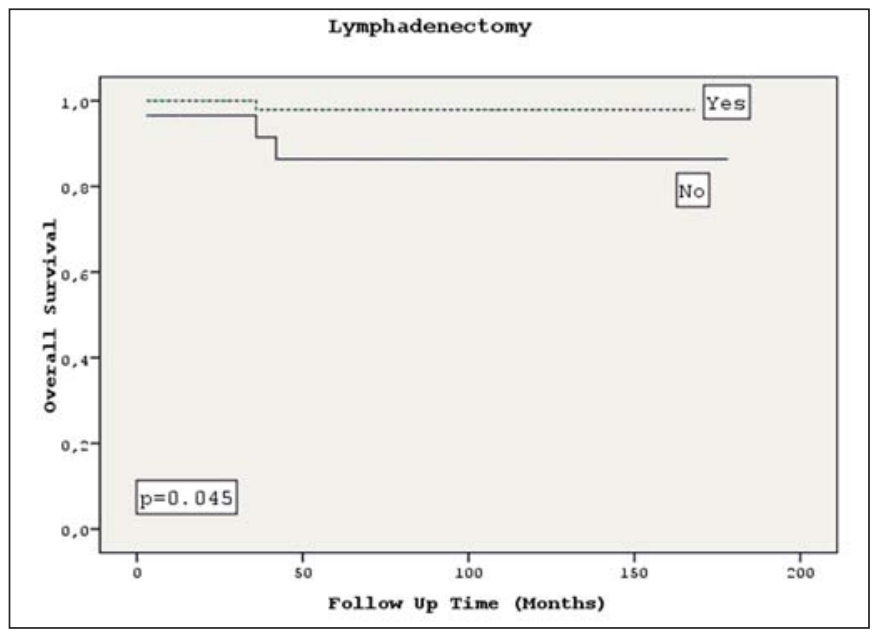

Figure 1: Survival effect of lymphadenectomy

Table 4: Distribution by adjuvant treatment for patients with grade 1 \& 2 tumor

\begin{tabular}{lccc}
\hline \multirow{2}{*}{ Lymphadenectomy } & \multicolumn{2}{c}{ Adjuvant treatment, $\mathrm{n}(\%)$} & $p$ value \\
& No & Yes & \\
\hline No & $5(17.2)$ & $24(82.8)$ & 0.78 \\
Yes & $11(15.1)$ & $62(84.9)$ & \\
\hline
\end{tabular}

\section{Discussion}

2009 FIGO classification system identifies 2 subgroups of stage I according to the depth of myometrial invasion (IA: MI $<50 \%$ and $\mathrm{IB}: \mathrm{MI} \geq 50 \%$ ) (6). As low-risk patients that defined as stage IA and grade $1 \& 2$ have a negligible risk of lymphatic spread and recurrence, they can be treated with only hysterectomy $(4,19)$. However, management of the patients with MI greater than half of myometrial thickness (stage IB) is controversial $(15,16)$. Most of the studies about this issue are retrospective and include all histopathological subtypes together. Furthermore, the performance of lymphadenectomy varies between these studies.

Histological subtype and grade, tumor size, LVSI, MI and stage were shown as prognostic factors in various studies (1923). However, we only included the patients with stage IB ( $72 \%$ of which were surgically staged) and endometrioid type histology and did not found any association between LVSI and DFS or OS. Maybe, LVSI could predict higher stages or lymphatic spread in patients with apparently uterine confined disease but may not be a predictor of survival in patients with established negative nodes. Furthermore, although, DFS and OS tended to be lower in patients with higher grades, this was not statistically significant (Table 2). This may be explained by the fact that almost all patients with grade 3 tumor received adjuvant treatment and underwent lymphadenectomy compared to those with grade $1 \& 2$ tumor in whom adjuvant treatment or lymphadenectomy was omitted to a certain extent.

Adjuvant therapy for patients with early stage disease is tailored according to FIGO stage and compiled prognostic factors $(5,15,24-26)$. In low-risk patients, external beam radiation carries more risk than benefit and women treated with radiotherapy had more risk for developing secondary malignancies and had experienced a lower quality of life due to radiation toxicity (27).

A prospective randomized study (PORTEC-1) of patients with stage I endometrial carcinoma (patients with both grade 
1 tumor and $\mathrm{MI}<50 \%$ were excluded) revealed that postoperative external beam radiotherapy reduces locoregional recurrence but has no impact on overall survival when lymphadenectomy is not carried out. Control group had almost four-fold increased risk of recurrence compared to radiotherapy group, however, there is no significant difference in respect to OS (15). GOG 99 trial evaluated the effect of adjuvant pelvic radiation in patients with intermediate risk endometrial cancer which was defined as any degree of myometrial invasion and any degree of histological grade. Non-endometrioid subtypes were excluded and all patients had lymphadenectomy. Patients were subdivided into a group with high intermediate risk which was defined as those with one or more risk factors (moderate to poorly differentiated tumor, presence of lymphovascular invasion, and outer third myometrial invasion) according to their age in this study. This group accounted for nearly two-thirds of the recurrences and cancer-related deaths. Adjuvant radiotherapy decreased the risk of recurrence in this subgroup of patients ( $26 \%$ vs. $6 \%$ ) and those on the RT arm demonstrated a somewhat lower overall death rate which was not significant (25). Our study differs from PORTEC-1 and GOG 99 in some aspects; we included only patients with MI $\geq 50 \%$ whereas in PORTEC- 1 , patients with $\mathrm{MI}<50 \%$ were included if they had grade $2 \& 3$ tumors, no patient had lymphadenectomy and patients with non-endometrioid histopathology were included and distinct from GOG 99; our cohort consisted of patients all with $\mathrm{MI} \geq 50 \%$, almost all patients with poorly differentiated tumor received adjuvant treatment and $72.9 \%$ of patients had lymphadenectomy. We observed that half of the patients in our cohort who didn't have adjuvant radiotherapy had recurrence compared to $12 \%$ of those who had, even though it didn't reach a statistical significance and there was no difference in respect to OS. The absence of significance in respect to DFS may be due to the low number of patients who did not receive radiotherapy in our entire cohort (n; 23 vs. 109).

The role of comprehensive surgical staging in endometrial cancer remains controversial. Among different institutions, the extent of lymphadenectomy varies from lymph-node sampling alone in the patients who have a high risk for nodal metastases to complete lymphadenectomy in all patients with endometrial uterine cancer, irrespective of grade and depth of myometrial invasion (1). Knowledge of the presence or absence of nodal metastases might guide the treatment interventions in some patients through the survival effect of this guidance remains to be established $(7,28)$. In addition to the guidance, survival benefit of removing apparently benign lymph nodes in early stage endometrial carcinoma has been shown in several studies (29-31). Recently, SEPAL trial revealed a survival benefit of combined pelvic and para-aortic lymphadenectomy compared to pelvic lymphadenectomy alone in the treatment of endometrial cancer patients (32). On the contrary, MRC ASTEC trial could not show any therapeutic effect of systematic pelvic lymphadenectomy (5) and a more a recent study by Koskas et al., which included 66.210 patients, concluded that poor outcome associated with higher risk of lymph node metastasis, that was calculated according to a nomogram, is not counterbalanced by lymphadenectomy (9). In our study performance of lymphadenectomy was associated with a better OS for the subgroup of patients with grade $1 \& 2$ tumors $(\mathrm{p}=0.04)$, although it was not valid for the entire cohort. In this subgroup $(n=102) 73$ patients had lymphadenectomy and importantly, there was no difference in respect to adjuvant treatment between the patients with or without lymphadenectomy (Table 4). We couldn't evaluate the effect of lymphadenectomy for the patients with grade 3 tumor because only 4 patients did not undergo lymphadenectomy and only 1 patient did not have adjuvant treatment.

In conclusion, adjuvant radiation seemed beneficial for prevention of recurrence as half of the patients who did not have any adjuvant radiotherapy recurred compared to $12 \%$ of patients those with adjuvant treatment. This needs to be confirmed by further studies which will include more patients who don't have adjuvant radiation. Lymphadenectomy had survival benefit for the patients with myometrial invasion greater than a half of myometrial thickness.

:Acknowledgements: None

Conflict of Interest: The authors declare no conflict of interest. All authors declare that this work does not have any funding source.

\section{References}

1. Chan JK, Kapp DS. The role of complete lymphadenectomy in endometrioid uterine cancer. Lancet Oncol 2007; 8(9):831-41.

2. Maggino T, Romagnolo C, Landoni F, et al. An analysis of approaches to the management of endometrial cancer in North America: a CTF study. Gynecol Oncol 1998;68(3): 274-9.

3. Maggino T, Romagnolo C, Zola, et al. P. An analysis of approaches to the treatment of endometrial cancer in western Europe: a CTF study. Eur J Cancer 1995;31A(12): 1993-7.

4. Mariani A, Webb MJ, Keeney GL, et al. Low-risk corpus cancer: is lymphadenectomy or radiotherapy necessary? Am J Obstet Gynecol. 2000;182(6):1506-19.

5. group As, Kitchener H, Swart AM, et al. Efficacy of systematic pelvic lymphadenectomy in endometrial cancer (MRC ASTEC trial): a randomized study. Lancet 2009; 373(9658):125-36.

6. Pecorelli S. Revised FIGO staging for carcinoma of the vulva, cervix, and endometrium. Int J Gynaecol Obstet. 2009;105(2):103-4.

7. Benedetti Panici P, Basile S, Maneschi F, et al. Systematic pelvic lymphadenectomy vs. no lymphadenectomy in early-stage endometrial carcinoma: randomized clinical 
trial. J Natl Cancer Inst. 2008;100(23):1707-16.

8. De Wilde RL, Rafei A, Herrmann A. Can a lymphadenectomy be avoided in early-stage endometrial adenocarcinoma? Arch Gynecol Obstet. 2014;290(5):973-8.

9. Koskas M, Fournier M, Luton D, et al. Survival impact of lymphadenectomy stratified by nodal metastatic probability in endometrial cancer. Ann Surg Oncol 2014;21 (7):2376-82.

10. Anderson B, Connor JP, Andrews JI, et al. Obesity and prognosis in endometrial cancer. Am J Obstet Gynecol 1996 Apr;174(4):1171-8; discussion 8-9.

11. Bogani G, Dowdy SC, Cliby WA, et al. Role of pelvic and para-aortic lymphadenectomy in endometrial cancer: current evidence. J Obstet Gynaecol Res 2014;40(2):301-11.

12. Yost KJ, Cheville AL, Al-Hilli MM, et al. Lymphedema after surgery for endometrial cancer: prevalence, risk factors, and quality of life. Obstet Gynecol 2014;124 (2 Pt 1):307-15.

13. Creasman WT, Odicino F, Maisonneuve P, et al. Carcinoma of the corpus uteri. FIGO 26th Annual Report on the Results of Treatment in Gynecological Cancer. Int J Gynaecol Obstet 2006;95 Suppl 1:S105-43.

14. Colombo N, Preti E, Landoni F, et al. Endometrial cancer: ESMO Clinical Practice Guidelines for diagnosis, treatment, and follow-up. Ann Oncol 2013;24 Suppl 6:vi33-8.

15. Creutzberg CL, van Putten WL, Koper PC, et al. Surgery and postoperative radiotherapy versus surgery alone for patients with stage-1 endometrial carcinoma: multicentre randomized trial. PORTEC Study Group. Post Operative Radiation Therapy in Endometrial Carcinoma. Lancet 2000;355(9213):1404-11.

16. Ortoft G, Hansen ES, Bertelsen K. Omitting adjuvant radiotherapy in endometrial cancer increases the rate of locoregional recurrences but has no effect on long-term survival: the Danish Endometrial Cancer Study. Int J Gynecol Cancer 2013;23(8):1429-37.

17. Tangjitgamol S, Khunnarong J, Katanyoo K, et al. Patterns of Adjuvant Therapy for Endometrial Cancer: Single Institutional Experience in Thailand. Int J Gynecol Cancer 2015;6[Epub ahead of print].

18. Gadducci A, Cosio S, Landoni F, et al. Adjuvant treatment and analysis of failures in patients with high-risk FIGO Stage Ib-II endometrial cancer: an Italian multicenter retrospective study (CTF study). Gynecol Oncol 2014;134 (1):29-35.

19. AlHilli MM, Podratz KC, Dowdy SC, et al. Risk-scoring system for the individualized prediction of lymphatic dissemination in patients with endometrioid endometrial cancer. Gynecol Oncol 2013;131(1):103-8.

20. Bendifallah S, Canlorbe G, Arsene E, et al. French
Multicenter Study Evaluating the Risk of Lymph Node Metastases in Early-Stage Endometrial Cancer: Contribution of a Risk Scoring System. Ann Surg Oncol 2015 Jan 7 [Epub ahead of print] .

21. Bendifallah S, Canlorbe G, Raimond E, et al. External validation of nomograms designed to predict lymphatic dissemination in patients with early-stage endometrioid endometrial cancer: a multicenter study. Am J Obstet Gynecol 2015;212(1):56 e1-7.

22. Bendifallah S, Canlorbe G, Raimond E, et al. A clue towards improving the European Society of Medical Oncology risk group classification in apparent early stage endometrial cancer? Impact of lymphovascular space invasion. Br J Cancer 2014;110(11):2640-6.

23. Loizzi V, Cormio G, Lorusso M, et al. The impact of lymph vascular space invasion on recurrence and survival in patients with early stage endometrial cancer. Eur J Cancer Care (Engl) 2014;23(3):380-4.

24. Deleon MC, Ammakkanavar NR, Matei D. Adjuvant therapy for endometrial cancer. J Gynecol Oncol 2014;25 (2): $136-47$.

25. Keys HM, Roberts JA, Brunetto VL, et al. A phase III trial of surgery with or without adjunctive external pelvic radiation therapy in intermediate risk endometrial adenocarcinoma: a Gynecologic Oncology Group study. Gynecol Oncol 2004;92(3):744-51.

26. Nout RA, Smit VT, Putter H, et al. Vaginal brachytherapy versus pelvic external beam radiotherapy for patients with endometrial cancer of high-intermediate risk (PORTEC2): an open-label, non-inferiority, randomised trial. Lancet 2010;375(9717):816-23.

27. Kong A, Johnson N, Kitchener HC, et al. Adjuvant radiotherapy for stage I endometrial cancer. Cochrane Database Syst Rev 2012;4:CD003916.

28. Aalders JG, Thomas G. Endometrial cancer--revisiting the importance of pelvic and para aortic lymph nodes. Gynecol Oncol 2007;104(1):222-31.

29. Kilgore LC, Partridge EE, Alvarez RD, et al. Adenocarcinoma of the endometrium: survival comparisons of patients with and without pelvic node sampling. Gynecol Oncol 1995;56(1):29-33.

30. Cragun JM, Havrilesky LJ, Calingaert B, et al. Retrospective analysis of selective lymphadenectomy in apparent early-stage endometrial cancer. J Clin Oncol 2005;23(16):3668-75.

31. Lutman CV, Havrilesky LJ, Cragun JM, et al. Pelvic lymph node count is an important prognostic variable for FIGO stage I and II endometrial carcinoma with high-risk histology. Gynecol Oncol 2006;102(1):92-7.

32. Todo Y, Kato H, Kaneuchi M, et al. Survival effect of para-aortic lymphadenectomy in endometrial cancer 